\title{
Differenzialdiagnose der infektiösen posterioren Uveitis
}

\author{
Differential Diagnosis in Infectious Posterior Uveitis
}

Autoren

Institute

\author{
J. G. Garweg' ${ }^{1}$, C. Tappeiner ${ }^{2}$
}

${ }^{1}$ Berner Augenklinik am Lindenhofspital, Universität Bern, Schweiz

2 Universitätsklinik für Augenheilkunde, Inselspital, Universität Bern, Schweiz

\section{Schlüsselwörter \\ - infektiöse posteriore Uveitis \\ - Diagnose \\ - Pathophysiologie \\ - Toxoplasmose \\ - Bartonellose \\ - akute Netzhautnekrose \\ - PORN}

Key words

- infectious posterior uveitis

- diagnosis

pathophysiology

- toxoplasmosis

- bartonellosis

- acute retinal necrosis

- PORN

eingereicht 30.9 .2010

akzeptiert $\quad 30.11 .2010$

Bibliografie

DOI http://dx.doi.org/10.1055/

s-0031-1273219

Klin Monatsbl Augenheilkd 2011; 228: 268-272 @ Georg

Thieme Verlag KG Stuttgart.

New York · ISSN 0023-2165

Korrespondenzadresse

Prof. Dr. Justus G. Garweg

Berner Augenklinik am

Lindenhofspital

Bremgartenstraße 119

3012 Bern

Switzerland

justus.garweg@augenklinik-

bern.ch

\section{Zusammenfassung \\ $\nabla$}

Infektiöse Ursachen bilden die größte ätiologische Gruppe posteriorer Uveitiden. Als Einzeldiagnosen stehen die Toxoplasmose als infektiöse Erkrankung und der Morbus Behçet als nicht infektiöse Erkrankung an erster Stelle. Bei akuten Entzündungsprozessen immunkompetenter Patienten ist eine exakte Diagnose häufig infolge einer recht dichten Glaskörperinfiltration schwierig zu stellen. In diesen Fällen trägt die Beurteilung des Krankheitsverlaufs zur Differenzialdiagnose wesentlich bei. Virale Netzhautnekrosen verschlechtern sich typischerweise hochakut, Sehstörungen und klinische Symptome bei Morbus Behçet und Toxoplasmose nehmen innerhalb weniger Tage bis 2 Wochen zu, während andere Erkrankungen einen eher schleichenden Verlauf zeigen. Die Dauer der Erkrankung und systemische Grunderkrankungen sind bei der ersten okulären Manifestation häufig nicht bekannt. Grundsätzlich ist die Sehfunktion bedroht, wenn die Makula in den entzündlichen Prozess einbezogen ist, wenn häufige Rezidive zu einer Beteiligung der Makula führen, aber auch wenn sich Sekundärkomplikationen wie ein Makulaödem entwickeln. Bei Kindern muss außerdem an die Gefahr einer Amblyopie infolge entzündlicher Medientrübungen gedacht werden, insbesondere bei Glaskörpertrübungen. Deshalb ist eine rasche interdisziplinäre diagnostische Abklärung und darauf basierende Therapiestrategie erforderlich. Diese muss einerseits ätiologisch abgestützt sein, andererseits sollte sie rechtzeitig steroidsparende Immunsuppresiva und eventuell chirurgische Maßnahmen beinhalten.

\section{Abstract \\ $\nabla$}

Infectious aetiologies represent the largest entity in patients affected by posterior uveitis. As a single diagnosis, ocular toxoplasmosis ist the most frequently diagnosed infectious entity, wheres Behçet's uveitis represents the most frequently encountered immunologic aetiology. In acute posterior uveitis, an exact clinical diagnosis is sometimes prohibited by a dense vitreal infiltration. In this situation, the clinical course and progression of the disease may help in the differential diagnosis. Rapidly progressive instances such as viral retinal necrosis may be differentiated from cases of ocular toxoplasmosis and Behçet's disease, which tend to present in the office within one to two weeks of symptoms, and from other more slowly progressive diseases such as mycotic or nematode infections. The clinical picture at presentation is influenced by the duration of disease, underlying systemic conditions, and by the natural course of disease including the healing process and scare formation. Generally, visual function has to be assumed at risk, if a lesion is located close to the macula, if frequent recurrences increase the likelihood of macular involvement and, furthermore, if secondary complications affect the macula, e.g., macular oedema. In paediatric cases, the risk of amblyopia is frequently under-estimated, namely in the context of persistent dense vitreal infiltration. Therefore, a rapid interdisciplinary clinically tailored diagnostic access has to be reinforced in order to establish an early, targeted and etiologically based therapeutic strategy including steroid-sparing immunomodulating agents and possibly surgical alternatives. 


\section{Einleitung}

\section{$\nabla$}

Die posteriore Uveitis ist eine in der augenärztlichen Praxis eher seltene Erkrankung mit einer sehr breiten klinischen Differenzialdiagnose. Sowohl klinischer Befund als auch Verlauf sind bei infektiöser Ursache variabel. Posteriore Uveitiden können sich perakut bis über Monate schleppend manifestieren, wie die Beispiele von hochakuten und symptomatischen viralen Netzhautnekrosen einerseits und die sich typischerweise über Monate langsam hinziehenden Nematoden- und Pilzinfektionen andererseits zeigen. Genauso verläuft die Erkrankung nach Abheilung der akuten Phase variabel, von nie wieder bis häufig rezidivierend (z.B. Toxoplasmose-Retinochoroiditis). Einer raschen und richtigen diagnostischen Einordnung kommt besondere Bedeutung zu. Die frühzeitige Diagnose einer eventuell vorhandenen systemischen Grunderkrankung oder Disposition, die Differenzierung von autoimmunologischen und infektiösen Prozessen und eine darauf abgestimmte systemische Therapie sind in den meisten Fällen essenziell für die funktionelle Prognose [1-3].

\section{Pathophysiologischer Hintergrund}

$\nabla$

Das klinische Bild der infektiösen Uveitis ist das Resultat der Aktivität der zugrunde liegenden Infektion und der immunologischen Wirtsreaktion darauf. Die klinisch sichtbare Aktivität der Entzündung spiegelt also nicht zwingend die Aktivität des Erregers wider, sondern entspricht bei immunkompetenten Individuen vor allem der durch die Infektion ausgelösten (teils überschießenden) Aktivität des Immunsystems zur Kontrolle der Infektion [4].

Grundsätzlich kann jede systemische mikrobielle Infektion mit einer Augenbeteiligung einhergehen. Infektiöse Systemerkrankungen können aber auch indirekt an der Pathogenese einer nicht infektiösen, sondern immunvermittelten Augenerkrankung im Rahmen einer unspezifischen Immunstimulation beitragen. Dies wird häufig über unspezifische Abwehrmechanismen erreicht (z.B. Komplementsystem, „Toll-like“-Rezeptoren) [9]. Typische Beispiele dafür sind die durch Yersinien ausgelöste HLAB27-assoziierte Uveitis, Bartonella-henselae-assoziierte Augenveränderungen $[7,8]$ und möglicherweise auch die altersbedingte Makuladegeneration [5-9]. Dies erschwert nicht nur die Einschätzung der Erkrankung und ihres Verlaufs, sondern erfordert im Einzelfall auch eine gute Balance zwischen antiinfektiöser Therapie (Antibiotika) und entzündungshemmenden Medikamenten (Steroide, Immunsuppressiva). Aus diesem Dilemma hilft auch die Diagnostik nicht weiter, da der Nachweis der Erreger-DNA mittels PCR (Polymerase-Kettenreaktion) zwar die Anwesenheit des Erregers, nicht jedoch seine pathophysiologische Rolle an der akuten Erkrankung beweist. Auf der anderen Seite zeigt der Nachweis einer lokalen Antikörperproduktion über den sogenannten Goldmann-Witmer-Index (Vergleich der Antikörperspiegel zwischen Kammerwasser und Serum) eine lokale Immunaktivität. Bisher ist jedoch wenig bekannt, ob dies auch unspezifisch ausgelöst werden kann, und wie schnell die Immunaktivität nach Abklingen der Erkrankung wieder auf Normalwerte zurückkehrt [10].
Tab. 1 Differenzialdiagnose entzündlicher Netzhaut- und Aderhautläsionen beim immunkompetenten Patienten anhand des Verteilungsmusters der Entzündung.

\begin{tabular}{|lll|}
\hline fokal & diffus & vaskulitisch \\
\hline Toxoplasmose & Zytomegalievirus & Morbus Behçet \\
\hline Lues & Varizella-Zoster-Virus & Morbus Eales \\
\hline Tuberkulose & Herpes-simplex-Virus & Sarkoidose \\
\hline Borreliose & VKH-Syndrom & Kollagenosen \\
\hline Toxokariose & Morbus Behçet & \\
\hline Candida & Lues & \\
\hline Histoplasmose (POHS) & & \\
\hline
\end{tabular}

POHS = presumed ocular histoplasmosis syndrome; VKH = Vogt-KoyanagiHarada.

\section{Klinische Einordnung und Verlauf \\ $\nabla$}

Die Differenzialdiagnose nicht HIV-assoziierter, nekrotisierender, retinitischer und chorioretinitischer Veränderungen ist breit. So sollte vor einer diagnostischen Abklärung versucht werden, die Differenzialdiagnose anhand des klinischen Bildes möglichst einzugrenzen. Das Erscheinungsbild als fokaler, diffuser oder vaskulitischer Prozess weist in der klinischen Beurteilung auf ganz unterschiedliche Differentialdiagnosen hin ( $\bullet$ Tab.1) [11]. Typischerweise präsentiert sich die infektiöse posteriore Uveitis als fokale Chorioretinitis, z.B. bei der Toxoplasmose, Lues, Tuberkulose, Borreliose, Toxokariose oder Histoplasmose. Im Gegensatz dazu verursachen virale Erkrankungen, welche vor allem die Neuroretina betreffen, keine klar abgrenzbare chorioretinale Läsion, sondern eine diffuse Netzhautnekrose ohne wesentliche choroidale Beteiligung. Differenzialdiagnostisch ist dies allerdings nicht immer von einer autoimmunen, vaso-okklusiv bedingten Netzhautnekrose im Rahmen eines Morbus Behçet, dem Vogt-Koyanagi-Harada(VKH)-Syndrom, einer atypischen Lues-assoziierten Uveitis oder einer aggressiven Verlaufsform einer erworbenen Toxoplasmose abgrenzbar. Eine primäre oder vorwiegend vaskulitische Manifestation weist meist auf eine Autoimmunerkrankung der Netzhaut hin, wobei der Morbus Behçet, die Sarkoidose (Morbus Boeck), Kollagenosen und der Morbus Eales abzugrenzen sind $[12,13]$.

Neben der Präsentation der Läsion spielen die Dauer der Symptome und die Progression der Erkrankung für die differenzialdiagnostische Einordnung eine zentrale Rolle. Für die Progression spielen einerseits die Virulenz und die Fähigkeit des infektiösen Erregers, das Immunsystem zu stimulieren, andererseits die Dauer des Replikationszyklus des Erregers eine Rolle. Ein schnell replizierender Erreger wie das Herpesvirus wird sich rascher ausbreiten können als ein langsam replizierender Erreger wie z.B. ein Pilz. Ein Erreger, der Antigene besitzt, die das Immunsystem stark stimulieren, kann eine heftigere Immunreaktion auslösen als ein gut bekannter Erreger, der nicht zum ersten Mal auf das Immunsystem trifft. Und bei einem Erreger schließlich, dessen antigene Strukturen ähnlich den humanen Antigenen sind, kann auch eine chronische Immunreaktion induziert werden, die im weiteren Verlauf völlig unabhängig von der Aktivität des Erregers verläuft, wie dies im Fall der metaherpetischen Keratitis und vermutlich auch bei der HLA-B27-assoziierten Uveitis zu beobachten ist. Die antiinfektiöse Therapie tötet idealerweise den Erreger. Der Erfolg einer antiinfektiösen Therapie zeigt sich aber nur in jenen Fällen, in denen die ErregerReplikation und nicht die Immunreaktion das Hauptproblem darstellt (z.B. bakteriellen Keratitis, virale Retinitis bei Immun- 
defizienz). Wenn die durch den Erreger ausgelöste Immunreaktion das größere Problem darstellt, wird auch eine erfolgreiche antiinfektiöse Therapie den Verlauf der Erkrankung nicht sichtbar beeinflussen. Dies bedeutet aber nicht, dass die Therapie nicht wirkt oder nicht sinnvoll ist, wie sich am Beispiel der bakteriellen Endophthalmitis zeigen lässt: Auch die antibiotisch ausreichend behandelte bakterielle Endophthalmitis kommt oft nicht zur Ruhe, bevor der Glaskörpers entfernt ist, weil auch tote Bakterien als Antigene einen Stimulus für eine massive Immunreaktion bilden [14]. Die Fähigkeit zur Immunstimulation hängt nicht von der Vitalität des Erregers, sondern vor allem von der Präsenz seiner Antigene ab. Diese können auch lange nach ausreichender Behandlung noch eine Entzündungsreaktion unterhalten. Ein Beispiel hierfür ist die chronische Toxocara-induzierte Uveitis, wo die Erregerzysten in der Netzhaut in der Regel keine lebendigen Erreger mehr enthalten, aber chronisch Antigene freisetzen und so eine progressive Narbenbildung induzieren [15].

Ein weiterer wichtiger Parameter für die Differenzialdiagnose bei posterioren Uveitiden ist (insbesondere bei rezidivierenden Verläufen) die Art der Narbenbildung. Man unterscheidet Erkrankungen ohne Narbenbildung von solchen mit einer klar abgegrenzten chorioatrophen, hellen Narbe mit häufig unregelmäßig pigmentiertem Randsaum und von Narben ohne jegliche Pigmentierungstendenz. Zur Gruppe der Erkrankungen ohne Narbenbildung gehört unter anderen die Bartonella-henselae-assoziierte „Neuroretinitis“ im Rahmen der Katzenkratzkrankheit und die HIV-assoziierte Mikroangiopathie. Eine diffuse Narbenbildung findet sich typischerweise bei Immunprozessen sowie bei früh erkannten und behandelten herpetischen Netzhauterkrankungen wie der PORN (progressive outer retinal necrosis). Eine chorioretinale Narbe ist der typische Befund für eine infektiöse Ursache (z.B. Toxoplasmose, Borreliose, Lues und Tuberkulose). Differenzialdiagnostisch muss gelegentlich auch die Aderhaut-Beteiligung bei Sarkoidose als nicht infektiöse Ursache für eine chorioretinale Narbe erwogen werden. Langfristige Aufhellungen ohne Pigmentierung beobachtet man vor allem im Rahmen der Birdshot-Uveitis als Folge der fokalen Pigmentatrophie ohne neuroretinalen Schaden [16-18] ( Tab.2).

Der Anteil an Kindern mit Uveitis beträgt etwa 5-10\% der Patienten in den Uveitis-Sprechstunden von Tertiärzentren. Knapp die Hälfte dieser Kinder leidt an posterioren Uveitiden. Da Kinder kaum über Sehstörungen klagen, wird bei ihnen im Vergleich zu Erwachsenen die Diagnose oft mit erheblicher Ver- spätung gestellt oder ist ein Zufallsbefund. Immer muss daran gedacht werden, dass eine infektiöse Uveitis bereits kongenital vorhanden oder erst im Laufe des Lebens erworben worden sein kann. Die häufigsten infektiösen Ursache von kongenitalen posterioren Uveitiden sind die Toxoplasmose, Röteln, das Cytomegalie-Virus, andere Erregern der Herpes-Gruppe und heute sehr selten die Syphilis. Diese Erkrankungen werden unter dem mnemotechnischen Begriff TORCHS zusammengefasst [19-23].

\section{Spezifische Krankheitsbilder}

Die okuläre Toxoplasmose ist mit 50\% die häufigste Ursache von posterioren Uveitiden. Man geht heute davon aus, dass die Mehrzahl der Fälle die Folge einer im Laufe des Lebens erworbenen Infektion und nicht kongenitalen Ursprungs ist. Wenn eine kongenitale Infektion nicht bereits bei der Geburt gesichert wurde, lässt sich die kongenitale von der postpartal erworbenen Form klinisch nicht differenzieren. Eine Makula-Beteiligung liegt in ca. $50 \%$ der erworbenen und $20 \%$ der kongenitalen Toxoplasmose Uveitiden vor [24]. Eine beidseitige Affektion findet sich bei ca. 30\% der kongenitalen okulären Toxoplasmose-Fälle, von denen aber ein großer Teil keine beidseitige Visusbeeinträchtigung erleidet [25, 26]. Eine Gesichtsfeldeinschränkung liegt hingegen sehr viel häufiger vor, ist jedoch in der Mehrzahl der Fälle nicht alltagsrelevant [27].

Eine kongenitale Zytomegalie-Virus(CMV)-Infektion ist bei einer von 300 - 5000 Geburten zu erwarten. Deutlich seltener ist eine Augenbeteiligung, kann dann aber zu einer schweren Sehbehinderung führen. Eine Röteln-Retinopathie ist seit der routinemäßigen Röteln-Impfung junger Mädchen sicherlich eine Rarität. Im Gegensatz zu der oft subakut fortschreitenden viralen Retinitis bei immunkompromittierten Individuen verläuft die akute Netzhautnekrose (ARN, acute retinal necrosis) bei immunkompetenten Patienten in der Regel perakut und desaströs: Rasch entwickeln sich infolge der ausgedehnten Netzhautnekrose eine Optikusatrophie und Netzhautablösung. Darum sollte bei Verdacht auf eine ARN oder eine andere visusbedrohende infektiöse Uveitis eine Kammerwasserdiagnostik durchgeführt und noch vor der Diagnosebestätigung eine auf die klinischen Verdachtsdiagnose gestützte, empirische antivirale oder antibiotische Therapie, gegebenenfalls in Kombination mit Steroiden, begonnen werden [28, 29]. Lässt sich die Diagnose über die Kammerwasser-Analyse nicht bestätigen, kann im Einzelfall auch eine Vi-

Tab. 2 Differenzialdiagnose chorioretinitischer Läsionen anhand der Narbenbildung.

\begin{tabular}{|c|c|c|c|}
\hline keine Narbenbildung & $\begin{array}{l}\text { nicht abgrenzbare } \\
\text { Narbe }\end{array}$ & $\begin{array}{l}\text { atrophe Narbe mit unregelmäßig pigmentiertem } \\
\text { Rand }\end{array}$ & $\begin{array}{l}\text { diskrete spät aufhellende } \\
\text { Narbe }\end{array}$ \\
\hline MEWDS & AMPPE & Multifokale Choroiditis & $\begin{array}{l}\text { mit oder ohne aktive Verän- } \\
\text { derungen }\end{array}$ \\
\hline $\begin{array}{l}\text { Bartonella-assoziierte } \\
\text { „Neuroretinitis“ }\end{array}$ & serpiginöse Choroiditis & Choroidopathia interna punctata (PIC) & Birdshot-Uveitis \\
\hline $\begin{array}{l}\text { HIV-assoziierte Mikro- } \\
\text { angiopathie }\end{array}$ & $\begin{array}{l}\text { PORN (herpetische } \\
\text { Retinitis) }\end{array}$ & diffuse subretinale Fibrose & \\
\hline \multirow[t]{3}{*}{$\begin{array}{l}\text { akute vitelliforme } \\
\text { Makulopathie }\end{array}$} & & $\begin{array}{l}\text { Uveitis bei Toxoplasmose, Borreliose, Lues, Tbc, } \\
\text { Sarkoidose }\end{array}$ & \\
\hline & & $\begin{array}{l}\text { Choroiditis/Chorioretinitis bei Pneumozystis Infektion } \\
\text { und Mykosen }\end{array}$ & \\
\hline & & $\begin{array}{l}\text { sympathische Ophthalmie, VKH, bakterielle Uveitis } \\
\text { (Roth Spots) }\end{array}$ & \\
\hline
\end{tabular}

AMPPE = akute multifokale plakoide Pigmentepitheliopathie; MEWDS = multiple evanescent white dot syndrom; PIC = punctate inner choroidopathy; PORN = progressive outer retinal necrosis; VKH = Vogt-Koyanagi-Harada Syndrom . 
trektomie oder Biopsie von intraokularem Gewebe zur definitiven Diagnosesicherung gerechtfertigt sein [30].

Während die Borreliose eine eher überschätzte Ursache für infektiöse Uveitiden darstellt, wird die Bartonellose (Katzenkratzkrankheit) als Ursache häufig nicht ausreichend in Erwägung gezogen. Schätzungen des Centers for Disease Controll (CDC) gehen davon aus, dass 66 von 1 Mio. Menschen an einer Bartonellose leiden. Typischerweise handelt es sich um Kinder mit Katzenkontakt, was der Erkrankung den Namen gegeben hat [31, 32]. Es handelt sich um eine grippeähnliche Erkrankung mit Lymphknoten- und Speicheldrüsenschwellung im Gesicht und Halsbereich; gelegentlich wird eine typischerweise einseitige Visusminderung aufgrund einer Neuroretinitis und Papillitis beobachtet ( $\bullet$ Abb.2). Die Bartonellen-Serologie ist zu diesem Zeitpunkt meist noch negativ und wird erst 3-12 Wochen nach der Infektion positiv. Eine hohe Sensitivität ist hingegen durch die Bartonella-PCR oder Kultur aus Bioptaten erzielbar [33, 34]. Im klinischen Verlauf ist typischerweise eine Spontanremission ohne Therapie - und meist auch ohne Diagnosestellung - Ausdruck des gutartigen Verlaufs. Schwerere Verläufe werden jedoch bei Patienten mit Immundefekten beobachtet [31, 32]. Die Bartonellen-assoziierte Neuroretinitis ist vermutlich nicht direkt infektiös, sondern Ausdruck der infektinduzierten Immunreaktion, die zu einer milden Vaskulitis im Bereich der Papille und der peripapaillären Kapillaren führt. Dabei handelt es sich um eine leukozytoklastische Vaskulitis der prälaminären Arteriolen der Papille mit sekundärer Lipidablagerung in der plexiformen Schicht (Henle-Nervenfaserschicht der Makula), die zu der deskriptiven Einordnung als Neuroretinitis meist mit CircinataAtoll im Bereich der Makula geführt hat. In der Tat findet sich in der Netzhaut aber nur makrophagozytärer Abräumprozess in der äußeren plexiformen Schicht und keine Retinitis, sodass der

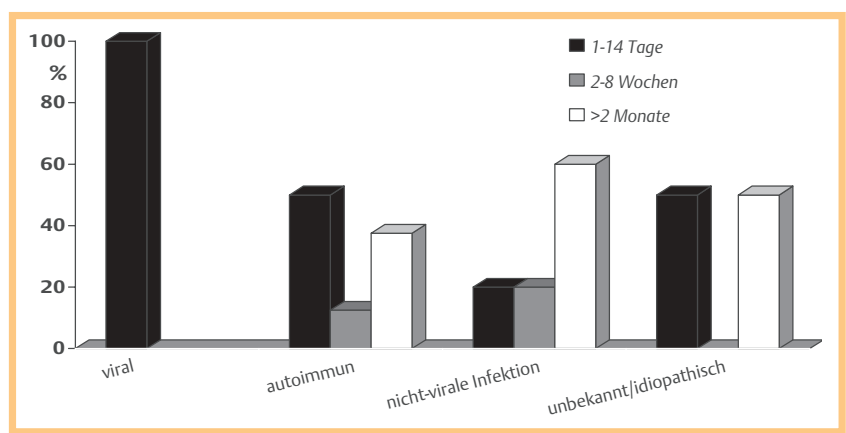

Abb. 1 Zeitlicher Verlauf der Uveitis als differenzialdiagnostischer Parameter aus einer eigenen konsekutiven Serie.
Ausdruck „Neuroretinitis“ eigentlich falsch ist [35-37]. Die Neuroretina selbst ist nicht entzündlich verändert, klinisch sieht man die Folgen der Schrankenstörung mit Ödem und Exsudaten, welche sich nach Abheilung komplett zurückbilden. Wenn klinisch kein Anhaltspunkt für eine Erregeraktivität vorliegt, hat die antibiotische Therapie bei dieser selbstlimitierenden Erkrankung vermutlich keinen Effekt und ist nur bei Patienten mit Immundefekt sinnvoll. Die Notwendigkeit einer Steroidtherapie ist umstritten, kontrollierte Therapiestudien gibt es wegen der Seltenheit der Erkrankung nicht [38].

Nicht nur selten diagnostiziert, sondern in unseren Breitengraden kaum zu sehen, sind okuläre Nematodeninfektionen. Dazu zählen die Toxokariose, die Onchozerkose, die diffuse unilaterale subakute Neuroretinitis (DUSN) und die Zystizerkose [39]. Diese Erkrankungen zeigen einen schleichenden, jedoch meist chronisch-progressiven Verlauf. Deshalb ist nicht nur eine korrekte Diagnose und antiinfektiöse Therapie, sondern häufig zusätzlich eine Steroidtherapie zur Kontrolle der Entzündungsreaktion erforderlich. So soll eine langsam fortschreitende Gewebszerstörung verhindert werden. Die klinische Erscheinung ist anfangs recht unspektakulär, jedoch von zunehmender Narbenbildung gekennzeichnet. Diese kann von der Hornhaut bis zur Aderhaut und Sehnerv sämtliche Gewebe im Auge betreffen ( Tab.3). Die am weitesten verbreitete Nematoden-Infektion am Auge ist die Toxokariose. Mit einem Anteil von deutlich unter $1 \%$ aller Uveitisfälle ist sie jedoch eine Ausschlussdiagnose und klinisch von anderen posterioren Uveitiden gut abzugrenzen. Oft findet sich langfristig eine Traktionsamotio oder Netzhautfalten, welche vom Toxokariose-Herd zum hinteren Pol ziehen [15]. Die beiden anderen, weltweit relevanten Nematoden-Infektionen, die Onchozerkose und Zystizerkose, spielen in unseren Breitengraden kaum eine Rolle. Ein erst in jüngerer Zeit vor allem in Südost- und Mittelwest-Amerika beobachtetes uveitisches

Tab.3 Okuläre Nematoden-Infektionen (nach [39]).

\begin{tabular}{|lll|}
$\begin{array}{l}\text { Nematoden- } \\
\text { Stamm }\end{array}$ & Parasit & klinische Manifestation \\
\hline Toxokariose & $\begin{array}{l}\text { Toxocara canis } \\
\text { Onchozerkose }\end{array}$ & $\begin{array}{l}\text { Choroiditis, Amotio } \\
\text { retionae }\end{array}$ \\
\hline DuSN & $\begin{array}{l}\text { Kolvulus } \\
\text { unbekannte } \\
\text { Nematode }\end{array}$ & $\begin{array}{l}\text { Neuroretintis } \\
\text { Uneitis }\end{array}$ \\
\hline Zystizerkose & Taenia solium & $\begin{array}{l}\text { ant. Uveitis/Optikus- } \\
\text { affektion }\end{array}$ \\
\hline
\end{tabular}

DUSN = Diffuse unilaterale subakute Neuroretinitis.
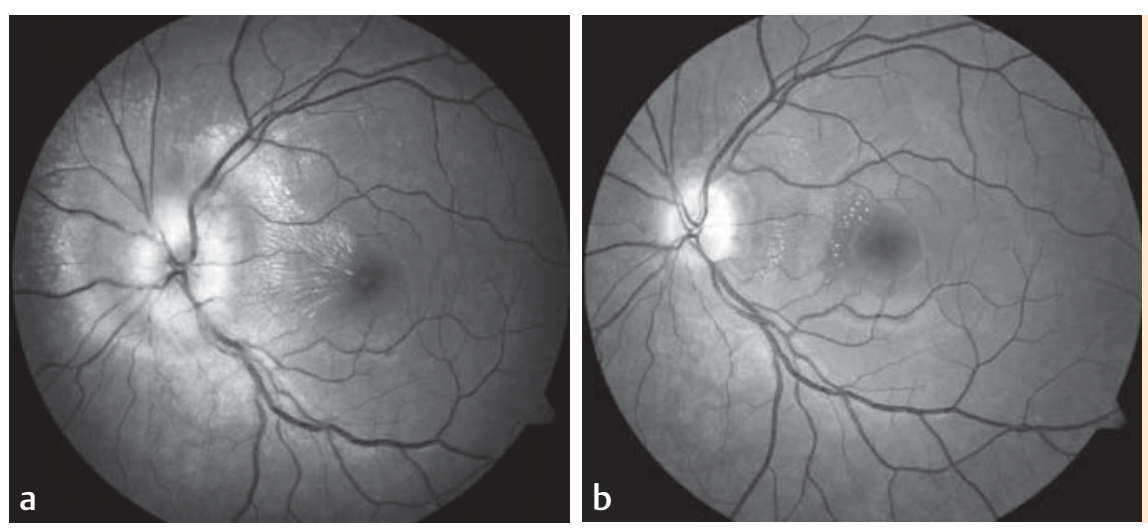

Abb.2 Bartonellen-assoziierte Neuroretinitis und Optikusneuritis bei einer 23-jährigen Patientin. Befund vor Therapie (oben) und 16 Wochen nach Steroidtherapie (unten). Typischerweise findet sich keine retinale Vaskulitis oder Glaskörper-Infiltration. 
Krankheitsbild ist die diffuse unilaterale subakute Neuroretinitis (DUSN), die immer häufiger auch außerhalb der Endemie-Gebiete beobachtet wird $(-$ Tab. 3 ).

Zusammenfassend bleibt festzuhalten, dass die Differenzialdiagnose infektiöser posteriorer Uveitiden sicherlich nicht immer einfach ist. Wenn man die klinische Präsentation und den Verlauf einbezieht, bleiben als häufigster Herdbefund die Toxoplasmose, als wichtigste flächige Erkrankung die virale Netzhautnekrose und als häufigste nicht infektiöse vaskulitische Erkrankung die Morbus-Behçet-Uveitis übrig. Diese Krankheitsbilder lassen sich meist recht gut gegeneinander abgrenzen und machen zusammen mehr als zwei Drittel der posterioren und Panuveitiden aus.

\section{Interessenkonflikt: Nein}

\section{Literatur}

1 Sudharshan S, Ganesh SK, Biswas J. Current approach in the diagnosis and management of posterior uveitis. Indian J Ophthalmol 2010; 58 : 29-43

2 Singh RP, Young LH. Diagnostic tests for posterior segment inflammation. Ophthalmol Clin 2006; 46: 195-208

3 Heiligenhaus A, Thurau S, Hennig $M$ et al. Anti-inflammatory treatment of uveitis with biologicals: new treatment options that reflect pathogenetic knowledge of the disease. Graefes Arch Clin Exp Ophthalmol 2010; 248: 1531-1551

4 Madigan WP, Raymond WR, Wroblewski KJ et al. A review of pediatric uveitis: Part I. Infectious causes and the masquerade syndromes. J Pediatr Ophthalmol Strabismus 2008; 45: 140-149

5 Robman L, Mahdi O, McCarty C et al. Exposure to Chlamydia pneumoniae infection and progression of age-related macular degeneration. Am J Epidemiol 2005; 161: 1013-1019

6 Guymer R, Robman L. Chlamydia pneumoniae and age-related macular degeneration: a role in pathogenesis or merely a chance association? Clin Experiment Ophthalmol 2007; 35: 89-93

7 Fukushima H, Numaga J, Yoshimoto $M$ et al. An ocular cat-scratch disease patient positive for cytoplasmic anti-neutrophil cytoplasmic antibody. Graefes Arch Clin Exp Ophthalmol 2001; 239: 882-885

8 Drancourt M, Bodaghi B, Lepidi H et al. Intraocular detection of Bartonella henselae in a patient with HLA-B27 uveitis. J Clin Microbiol 2004; 42: $1822-1825$

9 Chang JH, McCluskey PJ, Wakefield D. Toll-like receptors in ocular immunity and the immunopathogenesis of inflammatory eye disease. In, Br J Ophthalmol 2006; 90: 103-108

10 Garweg JG, Candolfi E. Immunopathology in ocular toxoplasmosis: facts and clues. Mem Inst Oswaldo Cruz 2009; 104: 211-220

11 McCannel CA, Holland GN, Helm CJ et al. Causes of uveitis in the general practice of ophthalmology. UCLA Community-Based Uveitis Study Group. Am J Ophthalmol 1996; 121: 35-46

12 Reddy UP, Albini TA, Banta JT et al. Post-streptococcal vasculitis. Ocul Immunol Inflamm 2008; 16: 35-36

13 Perez VL, Chavala SH, Ahmed M et al. Ocular manifestations and concepts of systemic vasculitides. Surv Ophthalmol 2004; 49: 399-418

14 Meier P, Wiedemann P. Endophthalmitis - clinical picture, therapy and prevention. Klin Monatsbl Augenheilkd 1997; 214: 175-191

15 Deuter CM, Garweg JG, Pleyer U et al. Ocular toxoplasmosis and toxocariasis in childhood. Klin Monatsbl Augenheilkd 2007; 224: 483-487
16 Verougstraete $C$. White spots syndromes. Bull Soc Belge Ophtalmol 2001; 279: 67-78

17 Quillen DA, Davis JB, Gottlieb JL et al. The white dot syndromes. Am J Ophthalmol 2004; 137: 538-550

18 Matsumoto Y, Haen SP, Spaide RF. The white dot syndromes. Compr Ophthalmol Update 2007; 8: 179-200

19 Patel H, Goldstein D. Pediatric uveitis. Pediatr Clin North Am 2003; 50: 125-136

20 Rosenberg KD, Feuer WJ, Davis JL. Ocular complications of pediatric uveitis. Ophthalmology 2004; 111: 2299-2306

21 Smith JA, Mackensen F, Sen HN et al. Epidemiology and course of disease in childhood uveitis. Ophthalmology 2009; 116: 1544-1551

22 Spital G, Heiligenhaus A, Scheider A et al. „White dot syndromes“ in childhood. Klin Monatsbl Augenheilkd 2007; 224: 500-506

23 Thadani SM, Foster CS. Treatment of ocular inflammation in children. Paediatr Drugs 2004; 6: 289-301

24 Bosch-Driessen LEH, Berendschot TTJM, Ongkosuwito JV et al. Ocular toxoplasmosis: clinical features and prognosis of 154 patients. Ophthalmology 2002; 109: 869-878

25 Wallon M, Kodjikian L, Binquet C et al. Long-term ocular prognosis in 327 children with congenital toxoplasmosis. Pediatrics 2004; 113: 1567-1572

26 Tan HK, Schmidt D, Stanford $M$ et al. Risk of visual impairment in children with congenital toxoplasmic retinochoroiditis. Am J Ophthalmol 2007; 144: 648-653

27 Scherrer J, Iliev ME, Halberstadt $M$ et al. Visual function in human ocular toxoplasmosis. Br J Ophthalmol 2007; 91: 233-236

28 Garweg J, Bohnke M. Varicella-zoster virus is strongly associated with atypical necrotizing herpetic retinopathies. Clin Infect Dis 1997; 24: 603-608

29 Garweg J, Fenner T, Bohnke $M$ et al. An improved technique for the diagnosis of viral retinitis from samples of aqueous humor and vitreous. Graefes Arch Clin Exp Ophthalmol 1993; 231: 508-513

30 Matos K, Muccioli C, BelfortJr R et al. Correlation between clinical diagnosis and PCR analysis of serum, aqueous, and vitreous samples in patients with inflammatory eye disease. Arq Bras Oftalmol 2007; 70: 109-114

31 Kodama T, Masuda H, Ohira A. Neuroretinitis associated with catscratch disease in Japanese patients. Acta Ophthalmol Scand 2003; 81: 653-657

32 Kelly PJ. A review of bacterial pathogens in Ctenocephalides felis in New Zealand. N Z Vet J 2004; 52: 352-357

33 Labalette P, Bermond D, Dedes V et al. Cat-scratch disease neuroretinitis diagnosed by a polymerase chain reaction approach. Am J Ophthalmol 2001; 132: 575-576

34 Kymionis GD, Siganos CS, Pallikaris IG. Late onset of serologic positive titers in a patient with Parinaud's oculoglandular syndrome. Semin Ophthalmol 2004; 19: 125-126

35 Suhler EB, Lauer AK, Rosenbaum JT. Prevalence of serologic evidence of cat scratch disease in patients with neuroretinitis. Ophthalmology 2000; 107: 871-876

36 Solley WA, Martin DF, Newman NJ et al. Cat scratch disease: posterior segment manifestations. Ophthalmology 1999; 106: 1546-1553

37 Ormerod LD, Dailey JP. Ocular manifestations of cat-scratch disease. Curr Opin Ophthalmol 1999; 10: 209-216

38 Conrad DA. Treatment of cat-scratch disease. Curr Opin Pediatr 2001; 13: $56-59$

39 Sabrosa NA, Zajdenweber M. Nematode infections of the eye: toxocariasis, onchocerciasis, diffuse unilateral subacute neuroretinitis, and cysticercosis. Ophthalmol Clin North Am 2002; 15: 351-356 